\title{
Evaluación de un Modelo Educativo Universitario: Una Perspectiva desde los Actores
}

\author{
Evaluation of a University Educational Model: A Perspective \\ from the Actors
}

Tiburcio Moreno Olivos*, Margarita Espinosa Meneses, Eska Elena Solano Meneses y María Magdalena Fresán Orozco

Universidad Autónoma Metropolitana-Cuajimalpa

\begin{abstract}
En el ámbito de las instituciones educativas la evaluación es considerada como un proceso esencial para la mejora continua. Son elementos de evaluación la administración, los alumnos, los docentes y también los modelos educativos bajo los cuales se desarrolla el proceso de enseñanza-aprendizaje. La evaluación de un modelo educativo permite identificar elementos clave que requieren atención inmediata para alcanzar las metas del aprendizaje. Esta investigación analizó el estado de apropiación del Modelo Educativo de la Universidad Autónoma Metropolitana, Unidad Cuajimalpa (UAMC), un modelo centrado en el aprendizaje que a diez años de su creación todavía no es conocido ni utilizado por la totalidad de los alumnos ni de los profesores. En este texto se exponen primeramente las concepciones de la evaluación y la importancia de realizarlas. Se describe el Modelo Educativo de la UAMC y el método utilizado para su evaluación. Posteriormente se presentan los factores que inciden en el alumnado y en el profesorado para su apropiación. Para la obtención de los datos del trabajo de campo se efectuó una investigación cuantitativa y cualitativa. Se identificó que la mayoría de los docentes y alumnos conocen las principales características del modelo educativo, pero pocos las asumen.
\end{abstract}

Palabras clave: Evaluación educativa, Eevaluación de modelos educativos, Modelos educativos universitarios, Aprendizaje centrado en el alumno, Educación superior.

In the field of educational institutions, assessment is considered an essential process for continuous improvement. Elements of assessment are the administration, the students, the teachers and the educational models under which the teaching-learning process develops. The assessment of an educative model allows identifying key elements that require immediate attention to reach learning goals. This research analyzed the status of ownership of the Educative Model of the Universidad Autónoma Metropolitana, Unidad Cuajimalpa (UAMC), a model focused on learning that ten years after its creation is not yet known or used by all the students or the teachers. In this text are firstly exposed the conceptions of assessment and the importance of realizing them. The Educative Model of the UAMC is described, as well as the method used for its assessment. Later on are presented the factors that influence the student body and the faculty for its ownership. To obtain fieldwork data a quantitative and qualitative research was conducted. It was detected that most of the teachers and students know the main characteristics of the educative model, but few assume them.

Keywords: Educational assessment, Assessment of educative models, University educative models, Student-focused learning, Higher education.

*Contacto: tmoreno@correo.cua.uam.mx

issn: 1989-0397

www.rinace.net/riee/

https://revistas.uam.es/riee
Recibido: $\quad 8$ de abril de 2016

$1{ }^{\text {a }}$ Evaluación: 1 de junio de 2016

Aceptado: $\quad 30$ de junio de 2016 


\section{Introducción}

Este artículo se deriva de la evaluación del Modelo Educativo de la Universidad Autónoma Metropolitana Unidad Cuajimalpa (UAMC), proceso que se llevó a cabo desde inicios del 2014 tras el punto de inflexión que esta institución vivió en el momento de su integración en un mismo edificio, dado que antes de esa fecha las Divisiones y carreras que conforman la Unidad Cuajimalpa ${ }^{1}$ se ubicaban en diversos espacios de la Ciudad de México, lo cual dificultó que la implementación del modelo fuera idónea y se realizara de manera plena e integral desde la creación de la Unidad Cuajimalpa en 2005.

La desintegración física de las Divisiones incidió en la falta de homologación de conceptos y criterios con que se estaba implementando el modelo educativo, situación percibida tras la integración de toda la comunidad a principios del 2014, pues la cercanía de las diferentes instancias permitió el contraste de las diversas interpretaciones que se tenían del modelo, al grado que se consideró oportuno generar un espacio para evaluar el nivel de conocimiento, apropiación e implementación que el Modelo Educativo tenía en las comunidades de las áreas que lo conforman.

Ello implicó una valoración de la efectividad de todos los elementos inmersos en el proceso enseñanza-aprendizaje. La complejidad de dicha evaluación estriba en los diferentes actores y circunstancias que se la determinan, así como la cuestión de interpretarla como un proceso dinámico, continuo y sistémico, enfocado hacia múltiples variables entre las que fueron consideradas: el rol del alumno, el rol del profesor, la visión de la institución, la estructura curricular y extracurricular, la oferta educativa, así como los cambios de las conductas apreciados en los actores mediante lo cual se verifican los logros adquiridos en función de los objetivos propuestos por el modelo educativo de la UAMC. En este marco surge la Comisión encargada de la evaluación de los factores mencionados.

La importancia de la presente investigación reside en que la evaluación posibilita el perfeccionamiento de la acción docente generando líneas de acción para subsanar áreas de oportunidad y con ello lograr los objetivos con los que fue diseñado. Así, el objetivo de este trabajo se desprende de los trabajos de la Comisión de Evaluación del Modelo Educativo de la UAMC: analizar el grado de conocimiento, comprensión y apropiación que el alumnado y el profesorado tienen del Modelo Educativo de la Unidad y con ello evaluar su congruencia, relevancia y pertinencia.

Finalmente, este documento, apoyado en los resultados de la evaluación llevada a cabo por la mencionada Comisión de Evaluación, constituye un fundamento acerca de los cuestionamientos y autocrítica que la institución ha liderado, así como la postura constructiva con la que los enfrenta. El documento se estructura en los siguientes apartados: I) fundamentación teórica, II) método, III) resultados y IV) discusión y conclusiones.

\footnotetext{
${ }^{1}$ La Unidad Cuajimalpa de la UAM está conformada por tres divisiones: División de Ciencias de la Comunicación y Diseño, División de Ciencias Naturales e Ingeniería y División de Ciencias Sociales y Humanidades.
} 


\title{
2. Fundamentación teórica
}

\subsection{Concepciones de evaluación en educación}

La evaluación es un concepto polisémico que ha sufrido una serie de transformaciones importantes a través del tiempo. Desde sus inicios a principios del siglo XX, en que la evaluación era concebida como sinónimo de medición, influida por las ideas del conductismo y el positivismo se aspiraba a hacer de esta una práctica "científica", para lo cual se requería del empleo de instrumentos de evaluación que fueran válidos, objetivos y confiables. En estas coordenadas, la evaluación, inserta en un paradigma tecnológicoracional de la educación, era vista como un proceso aséptico y neutral y el evaluador como alguien libre de valores. En las últimas décadas esta representación simplista de la evaluación ha sido superada (al menos en el plano teórico), entre otras razones, por la evolución del pensamiento en las ciencias sociales y el progreso de la investigación educativa, y ha ido ganando mayor legitimidad el paradigma cualitativo, que sitúa a la evaluación en una perspectiva epistemológica más acorde con la naturaleza de los fenómenos educativos.

En la actualidad se reconoce que la evaluación es una disciplina y un campo de investigación cuyas prácticas necesariamente tienen un marcado carácter social; son prácticas complejas, multirreferenciales y contextualizadas. Al tiempo que se destaca que la evaluación tiene un componente técnico-metodológico y otro ético-moral, siendo más importante este último (Nevo, 1997).

Existen tantas definiciones de evaluación como autores han escrito sobre el tema, pero en el lenguaje coloquial (Gimeno, 1995):

\begin{abstract}
Se otorga al verbo evaluar el significado de estimar, calcular, justipreciar, valorar, apreciar o señalar el valor, atribuir valor a algo. La operación de evaluar algo o a alguien consiste en estimar su valor no material. En la práctica cotidiana dominante, el significado de evaluar es menos polisémico: consiste en poner calificaciones a los alumnos y aplicar las pruebas para obtener la información a partir de la que se asignarán esas calificaciones. (p. 338)
\end{abstract}

Otra definición significativa es la que aporta Ralph Tyler (1986), que concibe la evaluación como el proceso de determinar hasta qué punto se están alcanzando realmente los objetivos educativos. Otra definición muy aceptada, que ha sido sugerida por investigadores prestigiados como Cronbach (1963), Stufflebeam (1969) y Alkin (1969), refiere a la evaluación como el mecanismo que permite proporcionar información para la toma de decisiones. En décadas pasadas se produjo un cierto consenso entre los evaluadores que consideran la evaluación como la valoración del mérito o de la valía (Eisner, 1979; House, 1980; Scriven, 1967; Stufflebeam, 1974) o como una actividad que comprende tanto la descripción como el juicio crítico (Guba y Lincoln, 1981; Stake, 1967). El Comité Conjunto para los Estándares de Evaluación (Joint Committee on Standars for Educational Evaluation) publicó una definición de evaluación como "la investigación sistemática de la valía o del mérito de un objeto” (Joint Committee, 1981, p. 12). Por su parte, Cronbach y sus colaboradores (Cronbach, 1982; Cronbach et al., 1980) rechazan abiertamente la naturaleza crítica de la evaluación y abogan por un planteamiento del evaluador como "un educador cuyo éxito ha de ser juzgado por lo que los demás aprenden" (Cronbach et al., 1980, p. 11) más que como "árbitro de un partido de béisbol" (p. 18), a quien se contrata para que decida quien actúa "bien" o "mal”. 
Mientras que para Nevo (1997) la evaluación educativa consiste en la recogida sistemática de información referente a la naturaleza y a la calidad de los objetos educativos, esta definición combina la descripción con el juicio crítico, pero hace una distinción entre ambos ya que son de naturaleza distinta. La descripción puede basarse en la recogida sistemática de datos y, así, dar como resultado información altamente objetiva. En cambio, el juicio crítico se basa en criterios que, en la mayor parte de los casos, están determinados por valores, normas sociales y preferencias personales de los individuos o entidades que financian la evaluación.

\subsection{La participación de los actores en el proceso de evaluación}

En esta investigación partimos de un enfoque de evaluación basado en la colaboración, en el que las partes implicadas participan activamente en el desarrollo de la evaluación y en todas las etapas de su puesta en práctica. Desde la génesis del proyecto se partió de una perspectiva de la evaluación como un proceso de construcción conjunta donde cuentan todos los puntos de vista de los participantes. Desde esta noción estaríamos aproximándonos a lo que en la bibliografía especializada se conoce con el concepto de evaluación participativa. No obstante, habría que ser cautos en su empleo porque se advierte que no se trata solo de una simple cuestión de participación, sino que aquella refiere a un proceso que conlleva un replanteamiento general de la práctica evaluativa a través de la cual los actores involucrados son responsables últimos de cómo se desarrolla la evaluación asegurándose de que las necesidades de todos ellos son reconocidas (Murillo e Hidalgo, 2015, p. 46). Se trata de un enfoque democrático en el que los participantes se convierten en sujetos de la evaluación y no en simples objetos, como sucede en un enfoque de evaluación tradicional. En el caso que nos ocupa, profesores y alumnos participaron de forma dinámica, reflexionando de manera individual y colectiva, expresando en un marco de libertad sus apreciaciones y valoraciones con respecto a la puesta en marcha y operación del modelo educativo de la Unidad.

De acuerdo con Murillo e Hidalgo (2015, pp. 46-47) el hecho de que los sujetos participen y aporten al proceso evaluativo conlleva múltiples beneficios, entre los cuales destacan:

$\checkmark$ Ayuda a identificar las necesidades reales de los participantes al ser ellos mismos los que exponen qué quieren y necesitan.

$\checkmark$ Aporta información valiosa sobre el proceso de enseñanza-aprendizaje.

$\checkmark$ Genera información útil del proceso de evaluación, mostrando aquello que ha funcionado y lo que hay que mejorar.

$\checkmark$ Empodera a los estudiantes.

$\checkmark$ A menudo da voz a aquellos que en muchas ocasiones no son escuchados.

$\checkmark$ Promueve el aprendizaje de estrategias y competencias que ayudarán a los estudiantes en su desarrollo integral.

$\checkmark$ Refuerza el autoconcepto de los estudiantes.

En este sentido, coincidimos con Cousins y Withmore (1998), citados por Murillo e Hidalgo (2015, p. 47), quienes distinguen entre dos tipos de participación complementarios: a) evaluación participativa práctica: implica a sus estudiantes en la toma de decisiones y la resolución de problemas y se trata de un enfoque que incluye los principios democráticos en su práctica evaluativa pero no tiene un compromiso explícito 
con el cambio social y b) evaluación participativa transformadora: se tiene como objetivo el logro del cambio social, empoderando a los participantes de la evaluación, y se pone especial atención en la inclusión de aquellos participantes tradicionalmente oprimidos.

\subsection{Importancia de la evaluación en el modelo educativo}

En una época de profundos e irreversibles cambios en todos los ámbitos, se mira a la educación y al conocimiento como un producto de gran valor. En este nuevo escenario la labor de los educadores debería ser el aprendizaje; sin embargo, el debate público sobre educación induce a pensar que la tarea consiste en "conseguir objetivos", "quedar en los primeros puestos de clasificación de las universidades", "obtener buenos resultados en los exámenes de admisión o de egreso" o "ser una universidad con un alto nivel de solicitudes de ingreso". Aunque es indudable que los resultados de los alumnos son extremadamente importantes, se considera que un enfoque tan estrecho es erróneo (Stoll, Fink y Earl, 2010).

Una de las ideas más relevantes de los últimos años consiste en aceptar que la evaluación no es un componente situado al final del proceso, ni solo un medio para valorar el logro de ciertos productos esperados, una vez que ha concluido el proceso o finalizado la puesta en marcha de un proyecto. La evaluación es un elemento que debe incluirse desde el diseño mismo de un programa o proyecto educativo y debe estar permeando todas las etapas del proceso formativo, para lo cual se propone una evaluación integrada e interactiva, con carácter formativo. Pero no se trata de reemplazar una evaluación sumativa o final por una evaluación formativa o de proceso (Scriven, 1967), pues ambas se consideran importantes. Se trata de no privilegiar (como generalmente ocurre) la evaluación sumativa por encima de la formativa, puesto que esta última es aún más valiosa toda vez que permite tomar decisiones oportunas para mejorar el proceso mientras este ocurre.

A estas alturas no cabe la menor duda de la importancia que tiene la evaluación en cualquier modelo educativo, pues a través de ella podemos identificar las fortalezas y debilidades de la adopción de determinadas políticas educativas, de la implementación del currículum escolar y de la efectividad de ciertas prácticas pedagógicas, entre otros. Sobre todo en una era en que la transparencia y la rendición de cuentas en el uso de los recursos públicos se han convertido en una exigencia en sociedades democráticas cada vez más vigilantes de cómo y en qué se invierten los impuestos de los ciudadanos.

En la actualidad, la evaluación se ha convertido en una exigencia para los distintos niveles y estamentos del sistema educativo mexicano. Es habitual la aplicación de evaluaciones externas, internas o una combinación de ambas, aunque, desafortunadamente, no se suelen aprovechar los resultados o la información que genera la evaluación para tomar decisiones que redunden en una mejora de la educación pública.

Las universidades mexicanas, cada una de ellas con base en su autonomía, la visión y misión que se propone, diseña su propio modelo educativo y su estructura curricular, los cuales permanecen durante un tiempo y después son reemplazados por otros o bien rediseñados para ajustarlos a los cambios educativos y las necesidades sociales del contexto más amplio. Durante las últimas dos décadas, la mayor parte de las universidades e instituciones de educación superior del país han transitado hacia modelos educativos mucho más flexibles, integrales e innovadores. Uno puede consultar 
en Internet esos modelos educativos, compararlos e identificar su enorme isomorfismo. Claro, una cosa es lo que se declara en los documentos oficiales y otra muy distinta lo que ocurre en su implementación. Ya se sabe que ningún modelo educativo ni estructura curricular se aplica al pie de la letra, que hay una serie de mediadores que entran en juego, pero otra cosa bien distinta es cuando entre "el deber ser" y "el ser" aparece una brecha que resulta casi insalvable (Moreno, 2010).

Existen varias razones, pero sobre todo por las exigencias de organismos evaluadores y acreditadores del país (cuyos criterios e indicadores de evaluación suelen ser uniformes), las universidades se han visto obligadas a tener que poner a tono sus modelos educativos y curriculares. Digamos que el cambio, más que ser producto de una necesidad interna, parece más bien una respuesta reactiva a presiones externas. Pero la situación no termina ahí: lo cierto es que, salvo algunas excepciones, prácticamente la totalidad de las universidades mexicanas, una vez que implementan sus modelos educativos y curriculares, no hacen un seguimiento ni una evaluación para conocer el impacto que han tenido o para saber en qué medida se lograron los objetivos esperados.

Evaluar la implementación de un modelo educativo es una tarea imprescindible porque solo a través de esta valoración podremos conocer la efectividad de la propuesta e identificar la distancia que existe entre lo que se pretendía y lo alcanzado y, sobre todo, porque esta información orienta la toma de decisiones para introducir ajustes o cambios oportunos. Sin embargo, en la investigación realizada se encontró que no existen antecedentes de esta envergadura sobre evaluaciones de modelos educativos de universidades públicas en México.

\subsection{La evaluación del Modelo Educativo de la UAMC}

La Universidad Autónoma Metropolitana (UAM) se creó en 1974 con la intención de establecerse en los cuatro puntos cardinales de la Ciudad de México. Las tres unidades fundadoras fueron Azcapotzalco, Iztapalapa y Xochimilco. La cuarta Unidad (Cuajimalpa) surgió treinta años después, en 2005, en el poniente de la Ciudad de México. Cada Unidad desarrolló un modelo educativo propio.

El Modelo Educativo de la Unidad Cuajimalpa se diseñó en los primeros meses después de su creación y se caracteriza, en su aspecto pedagógico, por impulsar un aprendizaje centrado en el alumno, más que en los conceptos impartidos por el maestro. En este contexto, se vuelve esencial el diseño de experiencias de aprendizaje, las cuales deben ser evaluadas, con el fin de retroalimentar la discusión y el trabajo de grupos docentes para la adecuada consecución de los fines del Modelo: formar estudiantes que aprendan a aprender.

Para lograr lo anterior, el trabajo en equipo, la investigación, el trabajo interdisciplinario y la resolución de problemas son actividades que el docente debe fomentar y con ello posibilitar que el alumno desarrolle la habilidad de evaluar argumentos y de interpretar evidencias y así forme un pensamiento crítico que lo lleve, entre otras cosas, a involucrarse en un proceso permanente de aprendizaje autodirigido.

Asimismo, se espera que los docentes utilicen las tecnologías digitales para la creación de experiencias nuevas de aprendizaje, con el fin de promover la construcción de conocimiento. Para ello, los docentes deben conocer y utilizar estas herramientas tecnológicas y apoyarse en ellas para la búsqueda de información, así como para la práctica de distintas alternativas de telecomunicación y participación de los estudiantes, 
lo que les permitirá actuar como guías y coordinadores del trabajo colectivo de los alumnos, en un proceso formativo caracterizado por los apoyos disponibles más modernos.

Por su parte, el papel de la institución en torno al Modelo consiste en proporcionar una estructura flexible que permita el desarrollo del alumno en diversos ambientes de aprendizaje. Por ejemplo, la movilidad estudiantil es un requisito dentro del Modelo de la UAMC, por lo que el alumno debe estudiar en otra institución pública o privada, nacional o extranjera con la cual la UAMC tenga convenio, al menos un trimestre. Ello porque se considera que la entrada y salida de los estudiantes en diferentes momentos de su formación facilita su exposición a contextos culturales y educativos diferentes, los cuales enriquecen sus perspectivas de análisis y su capacidad de articulación de propuestas.

Como se observa, el modelo educativo de la UAMC permea a la estructura curricular y a los procesos de enseñanza-aprendizaje. Esta organización puede describirse así:

\subsubsection{Estructura curricular}

Se propone la construcción de un currículo flexible, por áreas de formación, que permita la movilidad:

1. Movilidad vertical (materias no seriadas pertenecientes a trimestres diferentes).

2. Movilidad horizontal (materias optativas divisionales).

3. Movilidad entre planteles y universidades (materias optativas de movilidad de intercambio).

La estructura curricular pretende: a) promover en los estudiantes la disposición y la capacidad para involucrarse en el aprendizaje autodirigido durante toda su vida y b) aprender a aprender como un proceso que requiere de métodos y pautas de trabajo diferentes a la enseñanza basada en la clase magistral. Aprender a aprender implica el desarrollo de capacidades especiales en el manejo de la información, el desarrollo de una capacidad reflexiva y crítica que no tiene posibilidades de ocurrencia en la clase magistral.

\subsubsection{Procesos de enseñanza-aprendizaje}

Procesos promovidos por el docente y que tienen como objetivo:

$\checkmark$ Procurar que los procesos de enseñanza-aprendizaje desarrollen en los alumnos la capacidad de aprender a aprender, fomentando en ellos el gusto por el conocimiento.

$\checkmark$ Incorporar estrategias docentes en los programas de estudio que incidan en la articulación e integración del conocimiento de acuerdo con el carácter teóricopráctico y los niveles formativos de los alumnos.

$\checkmark$ Promover la integración del conocimiento mediante el trabajo colectivo de los alumnos en actividades coordinadas por sus profesores.

$\checkmark$ Procurar que el proceso de enseñanza-aprendizaje se enriquezca con los métodos y resultados de los proyectos de investigación que se realizan en las diferentes áreas.

$\checkmark$ Promover que los alumnos empleen tecnologías de información y comunicación 
para la discusión, análisis, adquisición y transmisión del conocimiento.

$\checkmark$ Promover la elaboración y uso de materiales didácticos que contribuyan al proceso de enseñanza-aprendizaje.

Esta propuesta considera: aprendizajes orientados a procesos cognitivos, es decir, a la generación de estructuras cognitivas y, por ende, al trabajo con actitudes ante el conocimiento y sus fines; aprendizajes que busquen el manejo de lenguajes específicos de los campos disciplinarios, tanto en las ciencias, como en las humanidades y las artes; y aprendizajes por problemas que orienten las acciones a un nivel de integración teórico, técnico y metodológico para la habilitación en las diversas prácticas en el campo profesional.

El Modelo de la UAM Cuajimalpa pretende, en concreto, formar estudiantes con educación humanística e interdisciplinaria que posean conocimientos científicos y habilidades creativas; todo ello mediante una formación flexible, innovadora y socialmente pertinente.

Después de una década de implementación del Modelo Educativo en la UAMC, se consideró muy importante hacer una evaluación global e integral de este (antes se habían hecho evaluaciones parciales) para valorar el alcance de los objetivos establecidos.

\section{Método}

Con la intención de conocer el nivel de conocimiento y apropiación del Modelo Educativo en la UAM Cuajimalpa, se llevó a cabo una investigación cuantitativa y una cualitativa, con el fin de contar con datos desde dos perspectivas.

\subsection{Investigación cuantitativa}

Para la investigación cuantitativa se diseñó una encuesta que fue enviada por correo a los docentes. El objetivo de esta fue registrar si el encuestado conocía las características del Modelo. La encuesta presentó una lista de 20 rasgos, algunos de los cuales caracterizan al Modelo Educativo y otros se alejan de él. Estos rasgos fueron: flexibilidad, rigidez, autonomia, aprendizaje memorístico, investigación, exámenes objetivos, interdisciplinariedad, autoritarismo, pensamiento crítico, clase tradicional, rigor académico, atención al alumno, práctica profesional, movilidad, solución de problemas, aprendizaje activo, sustentabilidad, participación, dependencia y trabajo en equipo. La idea fue que el encuestado seleccionara los conceptos y los colocara en diferentes recuadros según su opinión, ya fuera que los considerara cercanos o propios del Modelo Educativo o justo en la posición contraria: ajenos al Modelo Educativo. La encuesta fue enviada por correo a todos los docentes y se contó con las respuestas de 53 académicos, que representan el $25 \%$ de la planta existente. Se capturaron las encuestas asignando un valor de 2 a los rasgos identificados como más cercanos (primer bloque de cuatro casillas) y -2 a los rasgos considerados más lejanos (segundo bloque). Asimismo, en el segundo grupo de rasgos se asignó un valor de 1 para los rasgos más cercanos y un valor de -1 para los más lejanos. Los rasgos no seleccionados se consideraron neutros (valor 0). De esta manera, los rasgos más reconocidos como propios del Modelo Educativo por los alumnos y los profesores obtuvieron un puntaje mayor. Los totales obtenidos por cada uno de los rasgos (nivel de proximidad y lejanía) se graficaron. 
De la misma forma, la encuesta fue enviada a la totalidad de alumnos (1375) y se contó con la respuesta de 330 alumnos, es decir, el 24\% de la población total. Las respuestas fueron graficadas del mismo modo antes descrito; se otorgó 2 para los rasgos característicos del Modelo y -2 para las características ajenas a él.

\subsection{Investigación cualitativa}

En lo referente a la investigación de tipo cualitativo, se realizó una entrevista en su formato de grupos focales. La técnica de los grupos focales permitió el desarrollo de una entrevista grupal abierta semiestructurada, en donde se seleccionó un grupo de alumnos y profesores con el objeto de analizar el nivel de comprensión y aplicación del modelo educativo de la Unidad. Se definieron tres poblaciones de estudio por División Académica, lo que constituyó un total de 9 grupos focales, cuya selección de participantes se basó en los criterios siguientes:

1. Grupos de alumnos ubicados con alrededor del 50\% de créditos cubiertos de la carrera (dos alumnos por carrera).

2. Grupos de alumnos en la fase final de la carrera (tres alumnos por carrera).

3. Grupos de profesores (dos profesores por carrera), integrando a profesores con alta y baja aceptación del modelo educativo.

Se programaron tres grupos por División Académica y se dedicó un día para realizar la entrevista colectiva a los tres grupos de cada División Académica (en total alrededor de 45 alumnos entrevistados y 18 profesores). Se utilizó una cámara de Gesell para la realización de las entrevistas, un guion conformado por tres bloques de preguntas, con una duración aproximada de 20 minutos cada uno. Las preguntas del primer bloque indagaron el conocimiento de los alumnos o profesores del modelo educativo. El segundo bloque se orientó a explorar cómo se vive el Modelo Educativo, con el fin de evidenciar la apropiación o no del Modelo en la comunidad universitaria. En tercer lugar, se solicitó a los entrevistados que exploraran posibles respuestas a la pregunta: "si fuese posible cambiar algo del modelo educativo, ¿qué cosa cambiarían?”, esto con el fin de evaluar propuestas de mejora. Las entrevistas se transcribieron íntegramente para su posterior análisis.

\section{Resultados}

\subsection{Resultados de los profesores: Encuesta (cuantitativo)}

Los docentes destacaron el pensamiento crítico, la flexibilidad y la interdisciplinariedad como las características más asociadas al Modelo Educativo. Estos rasgos fueron seguidos del trabajo en equipo, la solución de problemas, el aprendizaje activo, la autonomía y la movilidad. Los resultados se observan en la figura 1. 


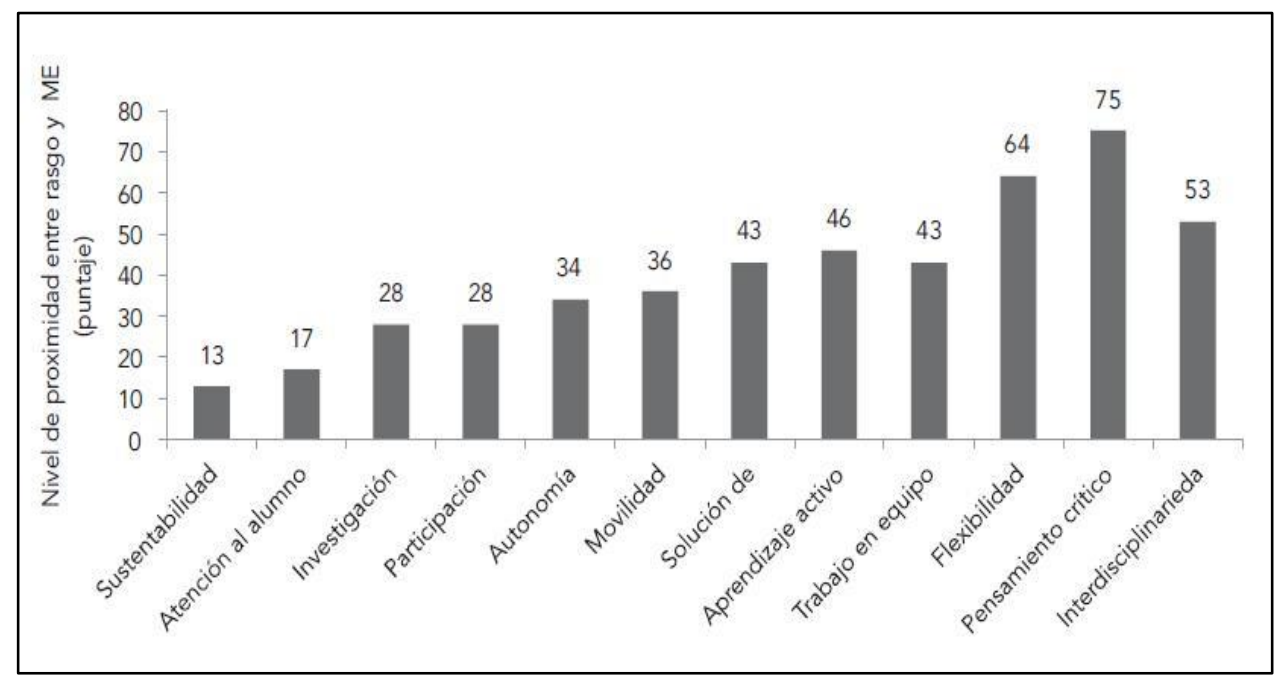

Figura 1. Resultados obtenidos con los docentes en la encuesta sobre el reconocimiento de las características asociadas con el Modelo Educativo

Fuente: Elaboración propia.

Por otro lado, los rasgos que los docentes menos asocian con el Modelo Educativo son: el aprendizaje memorístico, rigidez, autoritarismo, dependencia y clase tradicional. Con menor frecuencia fueron mencionados: práctica profesional, exámenes objetivos y rigor académico. Estos resultados se pueden apreciar en la figura 2.

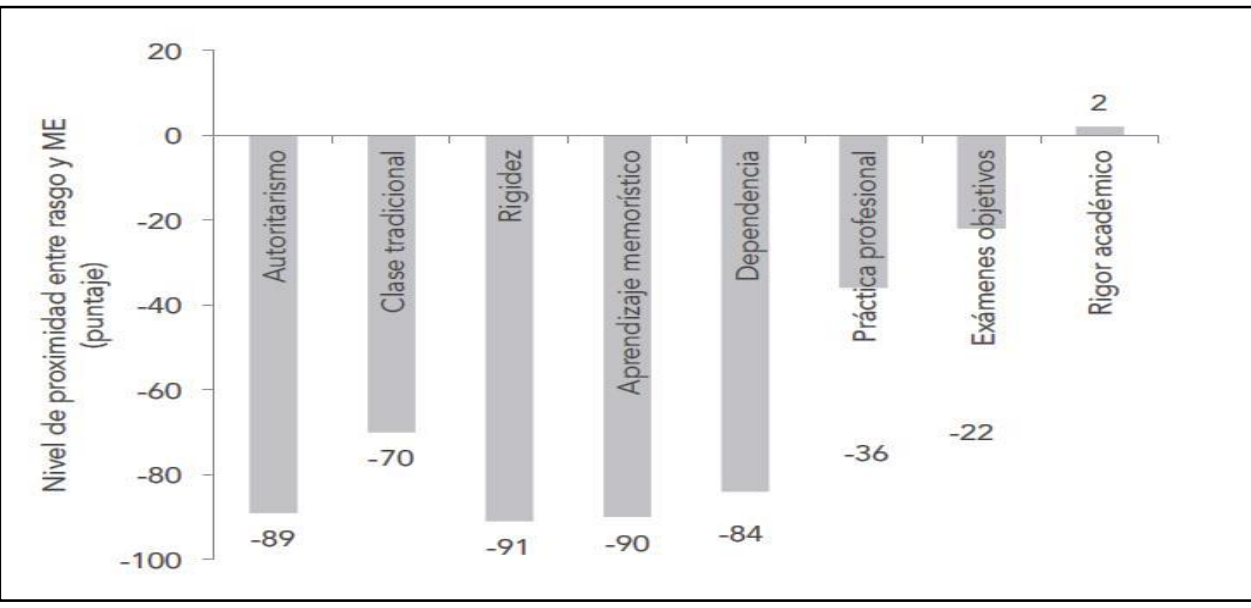

Figura 2. Resultados obtenidos con los docentes en la encuesta sobre el reconocimiento de las características no asociadas con el Modelo Educativo Fuente: Elaboración propia.

\subsection{Resultados de los profesores: Entrevista (cualitativo)}

La entrevista que se realizó a los docentes exploró los tres ámbitos mencionados: conocimiento del Modelo Educativo, cómo han aplicado el modelo y los elementos que modificarían con el fin de conducir de mejor manera el proceso de enseñanzaaprendizaje. Los datos obtenidos son los siguientes: 


\subsubsection{Conocimiento del modelo educativo}

La mayoría de los profesores que participaron en los grupos focales afirmaron conocerlo. La mayoría reconoce que el ME se inscribe en una corriente constructivista que está presente en la educación desde la década de 1980.

Las características que se mencionan como rasgos pertenecientes al Modelo son la interdisciplinariedad, la sustentabilidade y la responsabilidad social. En cuanto a los roles del docente y del alumno, señalan que el estudiante debe ser activo, crítico y constructor de su propio conocimiento. El docente debe ser más como un guía, un facilitador del aprendizaje y un diseñador de las experiencias educativas.

Los profesores afirmaron que conocieron los rasgos del modelo cuando ingresaron a la UAMC, mediante talleres o los documentos institucionales. Sin embargo, los profesores de más reciente ingreso desconocen la existencia del Modelo.

\subsection{2. ¿Cómo viven el Modelo Educativo?}

La serie de preguntas de este bloque buscaba inspeccionar si los docentes realizaban sus actividades a la luz del Modelo Educativo. En las respuestas se detectaron resistencias para su aplicación. Las razones expuestas pueden clasificarse en tres tipos: la infraestructura no es adecuada, los alumnos no responden y fallas en la preparación didáctica del docente, veamos:

a) La infraestructura de la Universidad no es la adecuada. La Red tiene fallas, el cañón es una herramienta necesaria para las clases (y no siempre se cuenta con él). También indican las ventajas de las clases tradicionales en grupos numerosos.

b) Los alumnos no están preparados para un modelo activo de aprendizaje y centrado en el estudiante porque "vienen de un sistema muy tradicional, donde el profesor tiene la verdad absoluta". También señalan que los conocimientos o actitudes que poseen los estudiantes no les permiten realizar un aprendizaje centrado en el alumno, pues:

¿Cómo diablos les enseñas, le das la autonomía, si no consolidan las herramientas más básicas?

Ningún alumno funciona, porque la mayoría de los estudiantes que tenemos no sienten placer en aprender, no están interesados, entonces, hacer que aprendan es difícil.

c) Los docentes tienen escasa preparación en cuestiones pedagógicas. Esta aserción representa una de las pocas autocríticas por parte de los docentes. Lo expresaron de la siguiente forma:

Creo que en ocasiones nos faltan herramientas pedagógicas para poder llevar a cabo, pues... ciertos ejercicios y, sobre todo, para poder guiar a los alumnos en ciertos sentidos.

Nosotros no nos formamos (creo que ninguno) a partir del modelo, y estamos más acostumbrados a enseñar contenidos que habilidades, a evaluar contenidos que a evaluar habilidades.

Una variante de este grupo de respuestas, en el que la crítica se ubica en el propio ámbito docente, la encontramos en las respuestas que reflejan la ideología del profesor con respecto a la funcionalidad y pertinencia de un modelo educativo, o bien lo rechazan porque lo perciben como algo impuesto, que ellos no eligieron. 
Esto ocasiona que algunos de los encuestados no crean en que un modelo pedagógico les ayude en su práctica docente, pues ellos creen que:

Todo buen profesor tiene su propio artificio y cada buen profesor es diferente de cualquier otro buen profesor.

Un modelo que se te impone no generaría lo mismo que un modelo que se sugiere, que es una posibilidad de crítica, de discusión sobre la pertinencia.

\subsection{3. ¿Qué cambiarían del Modelo Educativo?}

Este cuestionamiento obtuvo pocas respuestas. Los datos levantados pueden clasificarse en estos dos grupos:

a) Respuestas indirectas que se dirigen al ámbito de los alumnos, es decir, los problemas por los cuales no se puede aplicar el modelo están en el ámbito de los alumnos:

Nuestros estudiantes son niñas súper delicadas, súper deprimidas, o niños más deprimidos que las niñas.

El factor más grande no es el profesor, es el estudiante.

El modelo no funciona, mimar al alumno no funciona. Dar contenidos, aplicar exámenes y que muera el que tenga que morir, funciona. $Y$ si tiene que pasar 10 años, se va a quedar 10 años acá, entonces eso.

b) Respuestas concretas que apuntan a cambios específicos de los programas de estudio. Este grupo responde de forma directa a la pregunta realizada, aunque en número fueron pocas:

Introducir UE $A^{2}$ de carácter puramente motivacional, para cambiar actitud del estudiante.

Rediseñar el plan.

Hacer semestres en lugar de trimestres.

Es muy importante que no hay como trabajo colegiado docente..., que en otras instituciones sí hay reuniones al principio y al final del semestre donde expones tus métodos educativos, lo que te funciona y lo que no, y vas haciendo un aprendizaje colectivo...

\subsection{Resultados de los alumnos: Encuesta (cuantitativo)}

Los resultados obtenidos en las encuestas realizadas con los alumnos muestran que los rasgos más recordados entre los próximos al modelo educativo son: la investigación, el pensamiento crítico, la autonomía y el trabajo de equipo. Le siguen el aprendizaje activo, la flexibilidad y la interdisciplinariedad. Los elementos con menor asociación al Modelo (elegidos en un segundo momento) pero que también forman parte de él son: atención al alumno, participación, movilidad y solución de problemas.

Se graficaron de la siguiente forma (figura 3).

\footnotetext{
${ }^{2}$ En la Universidad Autónoma Metropolitana los cursos se designan con el nombre de Unidad de Enseñanza Aprendizaje (UEA).
} 


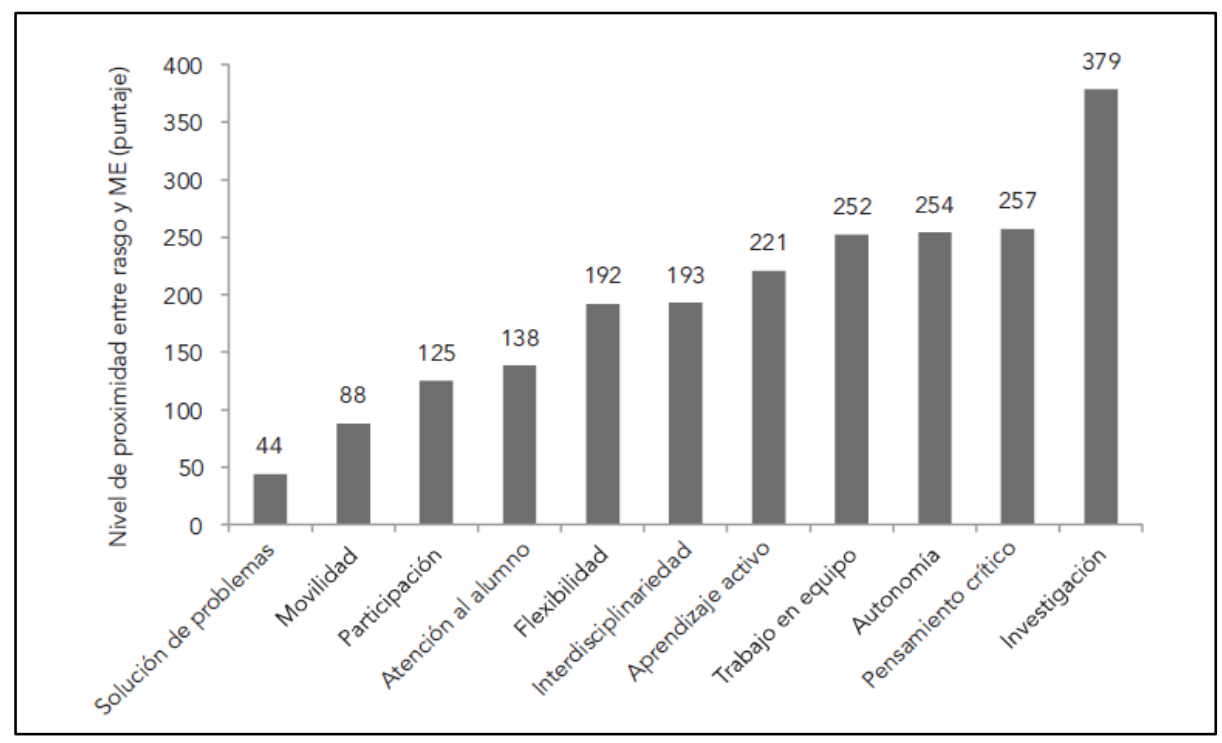

Figura 3. Resultados obtenidos con los alumnos en la encuesta sobre el reconocimiento de las características asociadas con el Modelo Educativo

Fuente: Elaboración propia.

Por su parte, entre los rasgos menos asociados con el Modelo Educativo están: autoritarismo, clase tradicional, dependencia, aprendizaje memorístico y rigidez. Con menor énfasis fueron mencionados rigor académico y práctica profesional. Las menciones a estos rasgos quedan representadas en la figura 4 .

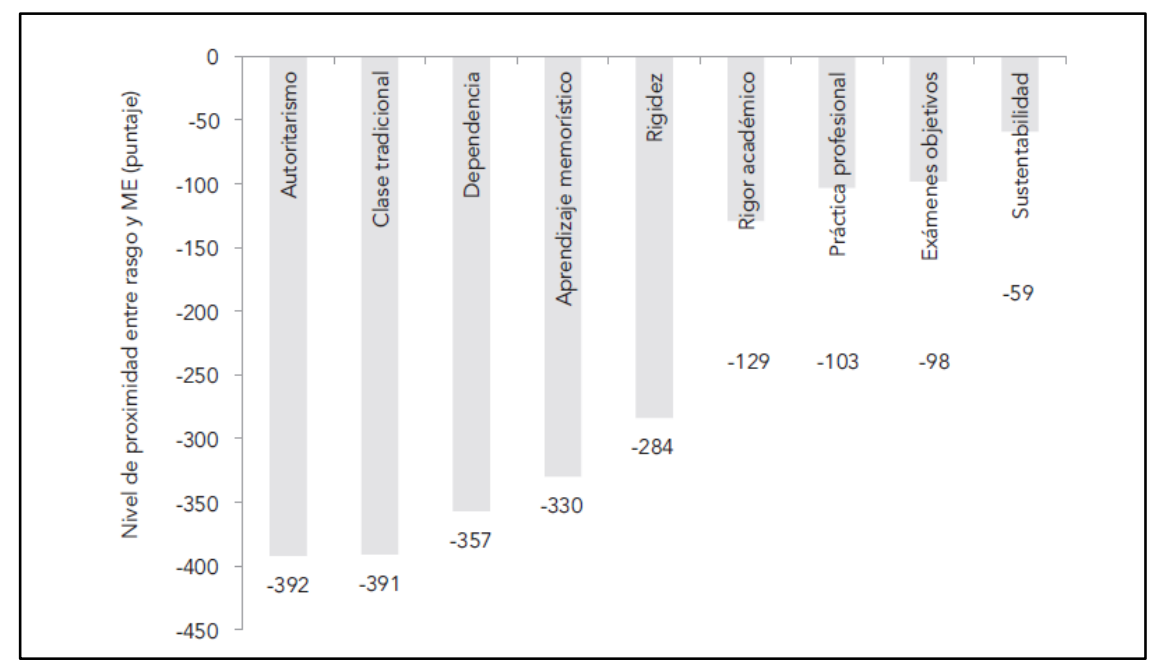

Figura 4. Resultados obtenidos con los alumnos en la encuesta sobre el reconocimiento de las características no asociadas con el Modelo Educativo Fuente: Elaboración propia.

Específicamente dos rasgos que corresponden al Modelo Educativo fueron señalados como no asociados a él, estos son: exámenes objetivos y sustentabilidad, es decir, los alumnos no identifican estas características como propias de la Unidad Cuajimalpa. 


\subsection{Resultados de los alumnos: Entrevista (cualitativo)}

Mediante los grupos focales se inspeccionaron dos niveles de comprensión del modelo educativo: conocimiento y apropiación. Entendemos por conocimiento aquella facultad mediante la cual una persona advierte la existencia, las cualidades y las relaciones entre elementos, mientras que con el término apropiación aludimos al proceso mediante el cual la persona obtiene para sí el conocimiento y también la experiencia generalizada que se tiene sobre un objeto determinado, material o mental. Vigotsky (1931, p. 103) señala que la apropiación de objetos de conocimiento está en función de una mediación ejercida por herramientas, que pueden ser físicas o simbólicas. Así, cuando hablamos de "apropiación del modelo educativo", hacemos referencia al hecho de que alumnos y docentes hayan interiorizado los rasgos del modelo, de tal manera que esos rasgos (autonomía, papel activo del estudiante, construcción y aplicación del conocimiento, etc.) se manifiesten eficazmente en los diversos contextos a los cuales los profesores y alumnos se enfrentan.

La inspección de estos dos niveles de comprensión del modelo arrojó los datos siguientes:

\subsubsection{Nivel de conocimiento del modelo educativo}

Los estudiantes, en su gran mayoría, conocen que la Unidad tiene un modelo educativo, nombran como rasgos de dicho modelo la interdisciplinariedad y el constructivismo. Sin embargo, no supieron explicar el significado de estos términos, estas son algunas de sus respuestas:

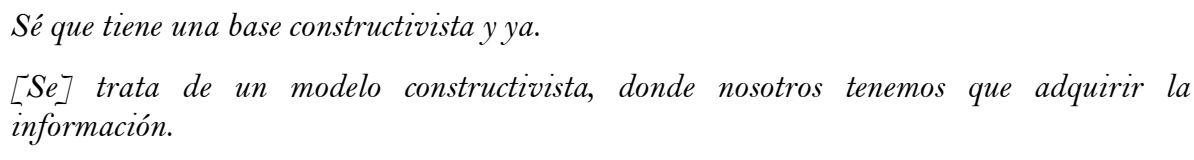

Otro de los puntos es la interdisciplinariedad, ¿no?

Otros rasgos que caracterizan al modelo fueron reconocidos solo por algunos de los estudiantes, los nombraron como: clases prácticas, mayor participación del alumno, un modelo centrado en la investigación.

[dentro del modelo, las clases] no son como estas cátedras tradicionales (...) sino es más
como una (...), por eso ponen un ejercicio, por eso hay un trabajo como que más práctico.
Que se basa en problemas, el profesor no nada más nos da la clase sino que espera que el
alumno también investigue por su parte, se informe y pueda aplicar los conocimientos a un
problema en específico.
El modelo de la UAM trata de romper e innovar el modelo tradicional de enseñar, está más
enfocado a la investigación.

\subsubsection{Nivel de apropiación del modelo educativo}

Mediante las entrevistas realizadas a los alumnos se trasluce que la apropiación del modelo no se ha dado en la mayoría de los estudiantes debido a diversos factores. Aquí presentamos los elementos que inciden en contra de la apropiación. Algunos de ellos propios de los estudiantes y otros como resistencias que los estudiantes perciben de los docentes.

a) Resistencias de los alumnos. Los estudiantes entrevistados tienen claro cuál es el rol del alumno en la UAMC, aunque no lo apliquen. Ellos declaran que el modelo fomenta estudiantes activos. Aluden a esta actitud con términos como 
[estudiantes] con iniciativa, autodidactas, responsables y sujetos activos. Estas fueron sus respuestas:

Ser más autodidacta y más una persona que se puede desenvolver de una manera más autónoma, más libre y más profesional.

No debe tomar un rol pasivo en el proceso sino debe cultivar el conocimiento, debe de cubrir lo que considere ya que no siempre se va a poder ver todo el temario y los temas que le interese y debe de sanar esas faltas.

Busca que seamos sujetos activos.

Capaces de desarrollar un pensamiento de actitud crítica, que seamos capaces de identificar problemas y plantear soluciones.

Sin embargo, afirman que la mayoría de sus compañeros, e incluso ellos mismos, no se desempeñan con la actitud de ser autodidactas, activos, responsables de su propio aprendizaje:

Yo creo que ser autodidacta es muy difícil porque necesitas tener hábitos propios, como decir "voy a leer, voy a invertir tanto tiempo en esto", pero el problema es que tengas tiempo también.

El alumno es muy difícil que cambie su iniciativa, sus hábitos de estudio, si no se lo inculca un profesor, la mayoría viene de escuelas rígidas, donde el profesor manda y dice, entonces los alumnos se consideran pasivos. Entonces ya el único que puede cambiar el modelo es el profesor, yo creo que es el único en el que está centrado es [en] el profesor, aunque el alumno se llame autodidacta pues ya son casos particulares...

Mis compañeros se sentaban, veían y decian "es que no entiendo", interrumpian al profesor a cada rato. Entonces yo creo que es donde falla mucho el modelo porque, aunque el profesor te diga "estudian en su casa", sabe que los alumnos no van a estudiar y otra vez tienen que reservar su clase para volver a explicar lo que no han aprendido. Sucede, ¿̇no?

Los entrevistados señalan específicamente factores que operan como resistencia: la inercia de venir de escuelas tradicionales, el hecho de que no se les ha enseñado a ser autodidactas y ello acarrea la irresponsabilidad.

b) Resistencias de los docentes. Se les cuestionó a los estudiantes cuál era el papel del docente en el modelo educativo de la UAMC. Lo primero que se destaca es que muchos de los entrevistados no supieron esta respuesta. Los que sí contestaron señalaron que el docente debe ser un facilitador, un guía, un tutor, con lo cual el alumnado tendría una participación más activa. Sin embargo, afirmaron que solo algunos de sus profesores asumen ese papel y que muchos de ellos no lo hacen:

La dinámica de interacción es muy tradicional, nuestro modelo es muy vanguardista por lo que hemos escuchado; sin embargo, en la práctica es aún muy difícil articular.

A mí me tocó que varios profesores no me fomentaban el que investigáramos. Llegábamos y... nos daban el curso y nunca nos dejaban que investigáramos, nunca se basaban en el Modelo Educativo.

El tener doctorado no les da habilidades docentes. Hay profesores que no tienen la motivación suficiente como para impartir a los alumnos los conocimientos.

Se observa en estas respuestas que los alumnos advierten la falta de herramientas pedagógicas en algunos docentes. Asimismo, aluden al desconocimiento del Modelo por parte de algunos profesores o bien afirman que no existe compromiso para asumirlo. 


\subsection{3. ¿Qué cambiarían para lograr mayores aprendizajes?}

El modelo de la UAMC incluye componentes curriculares (materias en el área de formación, movilidad estudiantil) y extracurriculares (idiomas, deportes, talleres). Sobre estos elementos, los alumnos entrevistados señalaron áreas que se deben reforzar, pues declaran que no operan de manera eficaz.

En cuanto a las materias interdivisionales, cuyo objetivo es el aprendizaje de la interdisciplina, los entrevistados afirmaron que son materias no relevantes para su formación. Afirman:

Ni me sirve lo que estoy aprendiendo, nada más vengo a calentar el asiento...

Con respecto a las materias que son interdivisionales, no digo que sean malas porque nos sirven para convivir con los demás, pero respecto a la carrera no tienen nada que ver con la carrera, nada, nada.

Por último, los entrevistados consideran que las actividades extracurriculares que ofrece la unidad deben tener mayor organización; en el área deportiva, exceptuando el fútbol, los demás deportes están desorganizados. Alegan que a los talleres (teatro, baile) les falta orden, sistematización y un lugar apropiado para realizarlos. En cuanto a las actividades culturales como las conferencias, señalan que están mal ubicadas, pues por lo regular se encima con sus horarios de clase.

Los entrevistados apuntan con estas respuestas a factores administrativos — los cuales corresponden a las autoridades de la universidad- que también juegan un papel importante en la apropiación del modelo por parte del estudiantado.

\section{Discusión}

Una de las primeras inconsistencias que se identificó es que las autoridades no han divulgado de forma eficaz lo que es el Modelo y los rasgos que lo caracterizan, pues se encontró que la mayoría de los entrevistados puede identificar sus características, pero algunas de ellas no saben exactamente a qué refieren. En este sentido, resulta indispensable que la universidad atienda la necesidad de socializar el modelo educativo, de manera que sea difundido tanto en alumnos como en profesores, así como contar con programas de formación docente que faciliten el trabajo de los profesores con los estudiantes. Para ello se requiere la revisión y mejora de las estrategias que hasta ahora se han implementado (cursos de inducción al modelo educativo sin continuidad, cuadernillos de difusión poco accesibles para toda la comunidad, etc.).

A partir del conocimiento impreciso que se tiene de algunos rasgos del Modelo y de las resistencias que los propios alumnos demuestran por ser ellos el centro del aprendizaje, y con esto estar obligados a ser más responsables, la apropiación del Modelo ha sido reducida en el alumnado.

La mayoría de los estudiantes conoce algunos rasgos del modelo (otros quedaron desdibujados o incluso no fueron mencionados, como la movilidad, el pensamiento crítico y la sustentabilidad), pero son pocos los que se asumieron como autodidactas, propositivos y acostumbrados a investigar. Así, desde la mirada de los estudiantes, los factores que inciden para que la apropiación del modelo no se haya logrado son principalmente: 1) el hecho de que la mayoría de los estudiantes provengan de escuelas tradicionales, donde no se impulsa la autonomía del estudiante; 2) el hecho de que 
algunos docentes carezcan de herramientas pedagógicas para crear los ambientes de aprendizaje que propicien la construcción de conocimiento; y 3) el hecho de que las autoridades de la universidad no organizan adecuadamente las actividades extracurriculares y con ello no favorecen la participación de los alumnos.

Por otro lado, los resultados evidenciaron de forma clara que los profesores, en su mayoría, sí conocen que el Modelo Educativo es de corte constructivista. Reconocieron y mencionaron características como: pensamiento crítico, interdisciplinariedad y trabajo activo como rasgos que lo constituyen. Los roles que desempeñan los docentes y los alumnos fueron descritos de acuerdo al Modelo, en el cual el proceso del aprendizaje está situado en el estudiante. Sin embargo, la aplicación de dicho Modelo no es uniforme y esto se debe a diversas razones. Desde la perspectiva de algunos docentes entrevistados, no ha habido mayor apropiación porque no existen las condiciones estructurales para ello y porque los estudiantes no tienen la preparación adecuada para asumirse como responsables de su propio aprendizaje. Pocos docentes fueron autocríticos al señalar que no cuentan con herramientas didácticas suficientes que les permitan diseñar ambientes de aprendizaje que posibiliten a los alumnos la construcción de saberes significativos. Nosotros consideramos al respecto que a los docentes les falta un mayor conocimiento del Modelo Educativo y el manejo de herramientas pedagógicas que les permitan diseñar sus actividades con los recursos con los que cuenta la Universidad. Consideramos al docente como la pieza clave para formar estudiantes que aprendan a aprender, de ahí la insistencia en que sea el profesor el primero en transformar la visión de su práctica pedagógica (Moreno, 2009).

Como se observa, la implementación del modelo educativo es un proceso simbiótico, que requiere la participación conjunta de profesores y alumnos: no se entiende que el solo cambio de actitud del alumno lo dirija hacia una gestión educativa, le haga independiente y le permita una autorregulación, para ello se requiere el diseño del curso del profesor donde se promuevan y dirijan las acciones hacia este cauce. Al mismo tiempo, se debe entender que el trabajo aislado del profesor sin las herramientas, preparación y condiciones necesarias resulta infructuoso. Ello deja evidencia de que, además de los dos actores analizados, el papel de la institución juega un rol crucial. A ella corresponde acercar los medios al docente, capacitarle sobre el manejo de recursos digitales, metodologías y estrategias de aprendizaje. Es la institución también la que ha de promover entre los profesores y alumnos, y de una manera más directa el modelo educativo: democratizar el rol que cada actor debe desarrollar, las ventajas que brinda el modelo educativo, a través de diversos y constantes instrumentos (digitales, escritos, gráficos) que permeen a todos los usuarios inmersos, ya que se ha observado que el primer paso para su implementación exitosa es el conocimiento tácito de dicho modelo. Una vez democratizado el modelo, será corresponsabilidad de profesores y alumnos el seguimiento de la operación de dicho modelo.

\section{Conclusiones}

La evaluación de cualquier modelo o proyecto educativo puede ser interna (permite valorar la calidad de la propuesta como tal), externa (permite valorar la eficacia en su aplicación) o ambas. En este caso, nosotros hemos realizado una evaluación externa, nos interesaba conocer el grado de conocimiento y apropiación del modelo educativo de la 
Universidad Autónoma Metropolitana, Unidad Cuajimalpa, por parte de profesores y alumnos, con el fin de proponer estrategias para que permitan que el modelo se vea reflejado en el proceso de enseñanza-aprendizaje, en el supuesto de que este hecho posibilita mayormente la formación de personas críticas, autodidactas y humanistas.

Los elementos que se consideraron en la evaluación del Modelo Educativo fueron: nivel de conocimiento y nivel de apropiación del modelo. Se documentaron resistencias de alumnos y docentes e identificaron elementos estructurales que operan en contra de la apropiación del Modelo Educativo de la UAMC.

En términos generales, consideramos que esta investigación resultó significativa pues permitió evaluar las acciones que se están realizando de forma adecuada y aquellas que deben reforzarse si se pretende que la Universidad trabaje en la formación de alumnos que aprendan a lo largo de su vida, así como pensar algunas posibles soluciones. Consideramos que esta investigación ofrece a las autoridades información rigurosa de la forma en que opera el Modelo Educativo en la UAMC a 10 años de su creación que, si bien resulta pertinente con las tendencias recientes de la educación superior en el mundo, resulta incongruente en la práctica.

Podríamos asegurar, por último, que hay un campo todavía por explorarse en lo que se refiere a la evaluación de modelos educativos. Por ello, la siguiente actividad de este grupo de investigación será caracterizar y contrastar los modelos educativos de otras universidades. Esto permitirá analizar sus logros y sus dificultades en torno al aprendizaje de los alumnos.

\section{Referencias}

Alkin, M. C. (1969). Evaluation theory development. Evaluation Comment, 2, 2-7.

Cronbach, L. J. (1963). Course improvement through evaluation. Teachers College Record, 64, 627683.

Cronbach, L. J. (1982). Designing evaluation of educational and social programs. San Francisco, CA: Jossey-Bass.

Cronbach, L. J., Ambron, S. R., Dornbusch, S. M., Hess, R. D., Hornick, R. C., Phillips, D. C., Walker, D. E. y Weiner, S. S. (1980). Toward reform of program evaluation. San Francisco, CA: Jossey-Bass.

Eisner, E. W. (1979). The educational imagination. Nueva York, NY: Macmillan.

Gimeno, J. (1995). La evaluación en la enseñanza. En J. Gimeno y Á. I. Pérez Gómez (Eds.), Comprender y transformar la enseñanza (pp. 334-397). Madrid: Morata.

Guba, E. G. y Lincoln, Y. S. (1981). Effective evaluation. San Francisco, CA: Jossey-Bass.

House, E. R. (1980). Evaluation with validity. Beverly Hills, CA: Sage.

Joint Committee on Standars for Educational Evaluation. (1981). Standars for evaluations of educational programs, projects, and materials. Nueva York: McGraw-Hill.

Moreno, T. (2010). El currículum por competencias en la universidad: Más ruido que nueces. Revista de la Educación Superior, 39(2), 77-90.

Moreno, T. (2009). La enseñanza universitaria: Una tarea compleja. Revista de Educación Superior, 38(3), 115-38. 
Murillo, F. J. e Hidalgo, N. (2015). Enfoques fundamentantes de la Evaluación de Estudiantes para la Justicia Social. Revista Iberoamericana de Evaluación Educativa, 8(1), 41-59.

Nevo, D. (1997). Evaluación basada en el centro. Un diálogo para la mejora educativa. Bilbao: Mensajero.

Scriven, M. (1967). The methodology of evaluation. En R. E. Stake (Ed.), AERA monograph series on curriculum evaluation. Chicago, IL: Rand McNally.

Stake, R. E. (1967). The countenance of educational evaluation. Teachers College Record, 68, 523540.

Stoll, L., Fink, D. y Earl, L. (2010). Sobre el aprender y el tiempo que requiere. Implicaciones para la escuela. Barcelona: Octaedro.

Stufflebeam, D. L. (1969). Evaluation as enlightenment for decision making. En W. H. Beatty (Ed.), Improving educational assessment and an inventory for measures of affective behavior (pp. 41-73). Washington, DC: National Education Association.

Stufflebeam, D. L. (1974). Meta-evaluation. Kalamazoo, MI: Western Michigan University Evaluation Center.

Tyler, R. (1986). Principios básicos del Currículum. Buenos Aires: Troquel.

Vigotsky, L. (1931). Historia del desarrollo de las funciones psíquicas superiores. Recuperado de http://www.colegiodepsicologosperu.org/w/imagenes/biblioteca/archivos/VygotskyObras-Escogidas-TOMO-3.pdf

\section{Breve CV de los autores}

\section{Tiburcio Moreno Olivos}

Doctor en pedagogía por la Universidad de Murcia. Miembro del Sistema Nacional de Investigadores (SNI) y del Consejo Mexicano de Investigación Educativa (COMIE). Integrante del Grupo Institucional de Investigación Educativa de la UAMC. Ha sido profesor en la Universidad de Quintana Roo y en la Universidad Autónoma del Estado de Hidalgo. En ésta última, fue Coordinador Académico del Doctorado en Ciencias de la Educación y Jefe del área Académica de Ciencias de la Educación, así como profesor en la Especialidad en docencia, en la Maestría y el Doctorado en Ciencias de la Educación. Ha impartido conferencias, seminarios y talleres de formación de profesores en diversas instituciones de educación superior del país y del extranjero. Líneas de investigación: evaluación del aprendizaje, currículum, y procesos de formación. E-mail: tmoreno@correo.cua.uam.mx

\section{Margarita Espinosa Meneses}

Doctora en Lingüística por la UNAM. Ha realizado investigación en el área de comunicación educativa y análisis del discurso. Ha participado en congresos nacionales e internacionales, entre sus colaboraciones están: "La argumentación como recurso para el aprendizaje colaborativo en internet: una propuesta"; "Análisis de las interacciones de estudiantes en un Foro de discusión”; “Análisis de los elementos que intervienen en el proceso de enseñanza virtual. Estudio de un caso específico”. Dentro de sus artículos 
publicados encontramos "Un acercamiento desde la comunicación educativa al uso de las TIC en la educación superior"; "Contextos educativos no formales: el museo y la apropiación del conocimiento científico"; "La educación on line. Evaluación de un curso virtual sobre escritura científica”. E-mail: mespinosa@correo.cua.uam.mx

\section{Eska Elena Solano Meneses}

Doctora en Diseño por la Facultad de Arquitectura y Diseño de la Universidad Autónoma del Estado de México con la tesis "Crítica Arquitectónica Sistémica: Un enfoque semiótico, cognitivo y simbólico", Maestra en Educación con Especialidad en Desarrollo Cognitivo por el Instituto Tecnológico y de Estudios Superiores de Monterrey Campus Toluca, Licenciada en Arquitectura por la Facultad de Arquitectura y Diseño de la Universidad Autónoma del Estado de México.

Actualmente es Investigadora de tiempo completo en la UAM-Cuajimalpa en el Departamento de Teoría y Procesos del Diseño. Sus investigaciones versan principalmente sobre la Educación, la Crítica del Diseño desde la perspectiva de la hermenéutica, la semiótica y la retórica. Asimismo se centra en el Diseño y la Ciudad en la Posmodernidad. E-mail: eskasolano@gmail.com

\section{María Magdalena Fresán Orozco}

Docente de pregrado y posgrado e investigadora desde 1975 en la Universidad Autónoma Metropolitana (UAM) institución que le otorgó recientemente el nombramiento de Profesora Distinguida. Es miembro del SNI. Fue Secretaria General de la UAM y Rectora fundadora de la Unidad Cuajimalpa. Coautora de: "Programas Institucionales Tutorías, Estudios de Egresados, Deserción, Rezago y Eficiencia Terminal y Evaluación del personal Académico". También es coautora de "Estructura Curricular", "Reflexiones sobre el modelo educativo de la UAM Cuajimalpa" y "Evaluación de la primera experiencia de movilidad" y "El Modelo educativo de la UAMC". En 2013 publicó "Acreditación del posgrado. Institucionalización e impacto en Argentina y México". Actualmente trabaja sobre la línea de investigación "La movilidad académica norte sur: su influencia en las élites científica, política y empresarial en México”. En 2014 recibió la Medalla Liderazgo Anáhuac en Educación. E-mail: mfresan@correo.cua.uam.mx 